\title{
Classificações de universidades mundiais, "Xangai" e outras
}

\author{
HERVÉ THÉRY
}

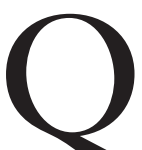

UEM SEGUE o debate sobre o futuro do ensino superior necessariamente ouviu falar da classificação de Xangai, a qual tem servido, cada vez mais, para medir o desempenho das universidades. Não era esse, no entanto, o objetivo da classificação, que foi inicialmente concebida para medir o atraso das universidades chinesas em relação às grandes universidades estrangeiras e de acordo com alguns observadores - para orientar a política chinesa de bolsas. Desde 2003, o Institute of Higher Education da Shanghai Jiao Tong University classifica anualmente as principais universidades mundiais em razão de seus "resultados de pesquisa", como muito claramente dizem os seus autores, e tem por única ambição produzir uma classificação "fundada sobre dados internacionalmente comparáveis e que qualquer um poderia verificar". ${ }^{1}$

Este artigo não é escrito por um especialista dos métodos de classificação das universidades, os leitores que querem saber mais sobre esse assunto poderão ler, por exemplo, os trabalhos recentes de Gingras (2008), Saisana \& D'Hombres (2008) ou Florian (2007) citados nas referências. Trata-se de uma contribuição às discussões sobre o tema apenas porque pareceu-nos que pode haver um aporte específico da cartografia a uma análise crítica das classificações: transferimos os resultados da classificação sobre um mapa-múndi² visando transformá-la numa geografia mundial das universidades, com seus pontos fortes e suas zonas de fraqueza.

Como essa classificação foi muito criticada, em razão de seus preconceitos e limitações, outras emergiram em vários países do mundo. Entre todas as que foram publicadas nos últimos anos, escolhemos três, após ter testado várias outras, por serem muito diferentes da classificação de Xangai, e entre si. Primeiro porque são estabelecidas por instituições de três países (França, Grã-Bretanha e Espanha), e sobre bases diferentes: a escola das Minas de Paris privilegia o sucesso dos ex-alunos no mundo dos negócios; o Times Higher Education (britânico) analisa - na medida do possível - a qualidade da formação dada aos estudantes; e por último, o laboratório Webometrics, do Consejo Superior de Investigaciones Científicas espanhol, mede a qualidade e a visibilidade dos $w e b$ sites das universidades.

\section{A “classificação de Xangai”}

O grupo que estabelece a classificação dita "de Xangai” (cujo nome oficial é Arwu, Academic Ranking of World Universities) é dirigido pelo professor Nian 
Cai Liu e inclui o Dr. Ying Cheng e o Sr. Linho Yang, do Institute of Higher Education da Shanghai Jiao Tong University. O grupo decidiu, desde junho de 2003, em decorrência de pedidos de colegas de diferentes países, publicar a sua classificação na web, e o site é consultado pelo mundo inteiro, com uma média de cerca de dois mil visitantes por dia. O grupo analisa todas as universidades cujos ex-alunos ou professores ganharam um Prêmio Nobel, uma medalha Fields (o equivalente ao Nobel na Matemática) ou tiveram artigos publicados na Nature ou Science, cujos pesquisadores figuram entre os mais citados em um determinado campo científico, bem como aqueles que possuem um número significativo de artigos indexados pelo Science Citation Index-Expanded (SCIE) ou Social Science Citation Index (SSCI). Ou seja, no total, mais de mil estabelecimentos, dos quais apenas os 500 primeiros são citados na lista publicada.

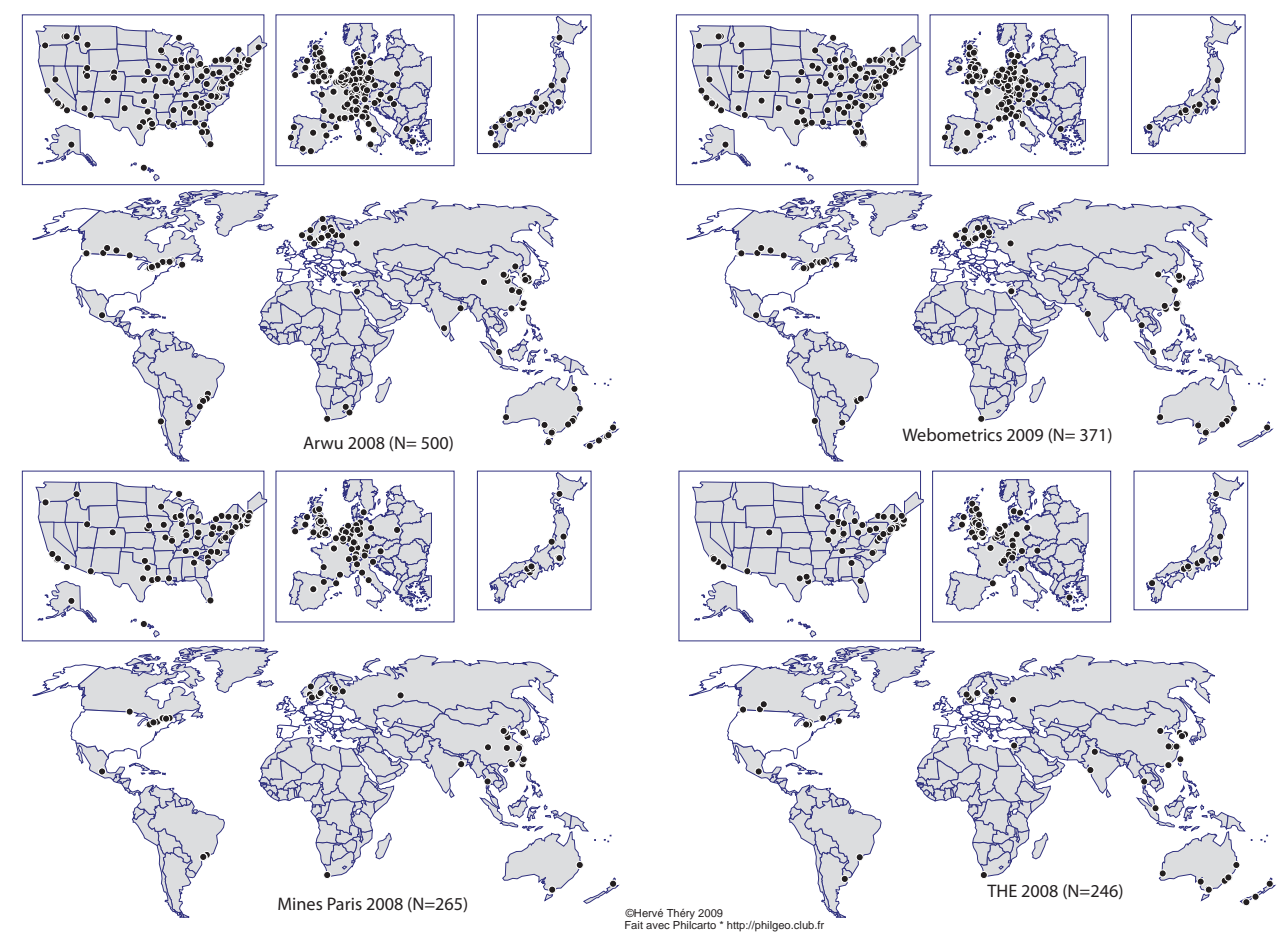

Figura 1 - As universidades analisadas pelas quatro classificações.

\section{Definição dos indicadores}

Seis indicadores foram selecionados, para cada um deles o estabelecimento mais bem classificado recebe a nota 100 , sendo a ordenação dos outros calculada em porcentagem dessa.

EX-Alunos (Alumni). A base de cálculo é o número de ex-alunos da Universidade (graduados e titulares de mestrado ou doutorado do estabelecimento) que receberam uma medalha Fields ou um Prêmio Nobel de Física, Química, Medicina e Ciências Econômicas. Pesos diferentes são atribuídos de acordo com 
os períodos de obtenção dessas distinções, dando mais ênfase aos mais recentes: $100 \%$ para os antigos alunos que o ganharam em 1991-2000, 90\% para 19811990, 80\% para 1971-1980, e assim por diante até 10\% para 1901-1910. Esse primeiro indicador estabelece uma nítida supremacia dos Estados Unidos e da Grã-Bretanha, particularmente bem representados entre os laureados do Prêmio Nobel, um fenômeno que tende a acentuar-se porque os antigos laureados são membros dos comitês que atribuem os prêmios.

PRÊMIOS (Award). A base é o número de funcionários de um determinado estabelecimento que ganharam um Prêmio Nobel, nos campos científicos onde existe, ou a medalha Fields em matemática, enquanto trabalhavam nele. Pesos diferentes também são atribuídos de acordo com os períodos em apreço: 100\% para os laureados de 2001-2006, 90\% para 1991-2000, 80\% para 1981-1990, 70\% para 1971-1980, até a 10\% para 1911-1920. Se um laureado é membro de vários estabelecimentos, os pontos obtidos são divididos entre eles.

CitaÇões $(\mathrm{HiCi})$. A base é o número de pesquisadores mais frequentemente citados nas disciplinas biológicas, na medicina, nas ciências físicas, na tecnologia e nas ciências sociais. A definição das categorias e os procedimentos detalhados podem ser encontrados sobre no site do Institute of Scientific Information (ISI Web of Knowledge).

NATURE e SCIENCE (N\&S). O indicador é constituído sobre o número de artigos publicados nas revistas Nature e Science entre 2002 e 2006. Um peso de $100 \%$ é atribuído quando a afiliação do autor corresponde ao seu principal lugar de trabalho, 50\% para uma segunda afiliação do autor, $25 \%$ para outras inscrições. Ambas as revistas publicam em inglês, Nature sendo publicada pelo Nature Publishing Group (com escritórios principais em Londres e Nova York) e Science pela American Association for the Advancement of Science, com sede em Washington. Não é surpresa alguma que os cientistas anglófonos tenham maior facilidade para publicar, não somente graças ao seu domínio do idioma, mas também porque os comitês científicos dessas revistas são majoritariamente compostos de investigadores procedentes desses países.

A Figura 2 mostra o resultado, para esse critério, das universidades selecionadas pelos autores do Arwu: a dimensão dos círculos é proporcional ao total de pontos Arwu e a sua cor ao critério N\&S.

SCIENCE CitATION INDEX (SCI). O cálculo se apoia sobre o número total de artigos classificados em Science Citation Index-expanded e Social Science Citation Index. Aqui também a predominância da língua inglesa é esmagadora e se re ete na classificação das universidades não anglófonas, como se vê claramente na Europa, onde as universidades britânicas dominam aquelas do continente.

Tamanho (Size). Para tentar reduzir os efeitos de dimensão, os pontos obtidos nos cinco indicadores anteriores são divididos pelo número de pessoas empregadas em tempo integral, ou o seu equivalente em tempo parcial. Para 2008, os números foram obtidos para os estabelecimentos situados nos Estados 
Unidos, no Reino Unido, na França, no Japão, na Itália, na Coreia do Sul, na Chéquia, na China, na Itália, na Austrália, na Holanda, na Suécia, na Suíça, na Bélgica, na Eslovênia, na Nova Zelândia etc.

Constata-se, contudo, que esse indicador produz pouco efeito, já que os melhores resultados são obtidos exatamente pelas grandes universidades do nordeste dos Estados Unidos e da Califórnia, assim como as universidades britânicas...

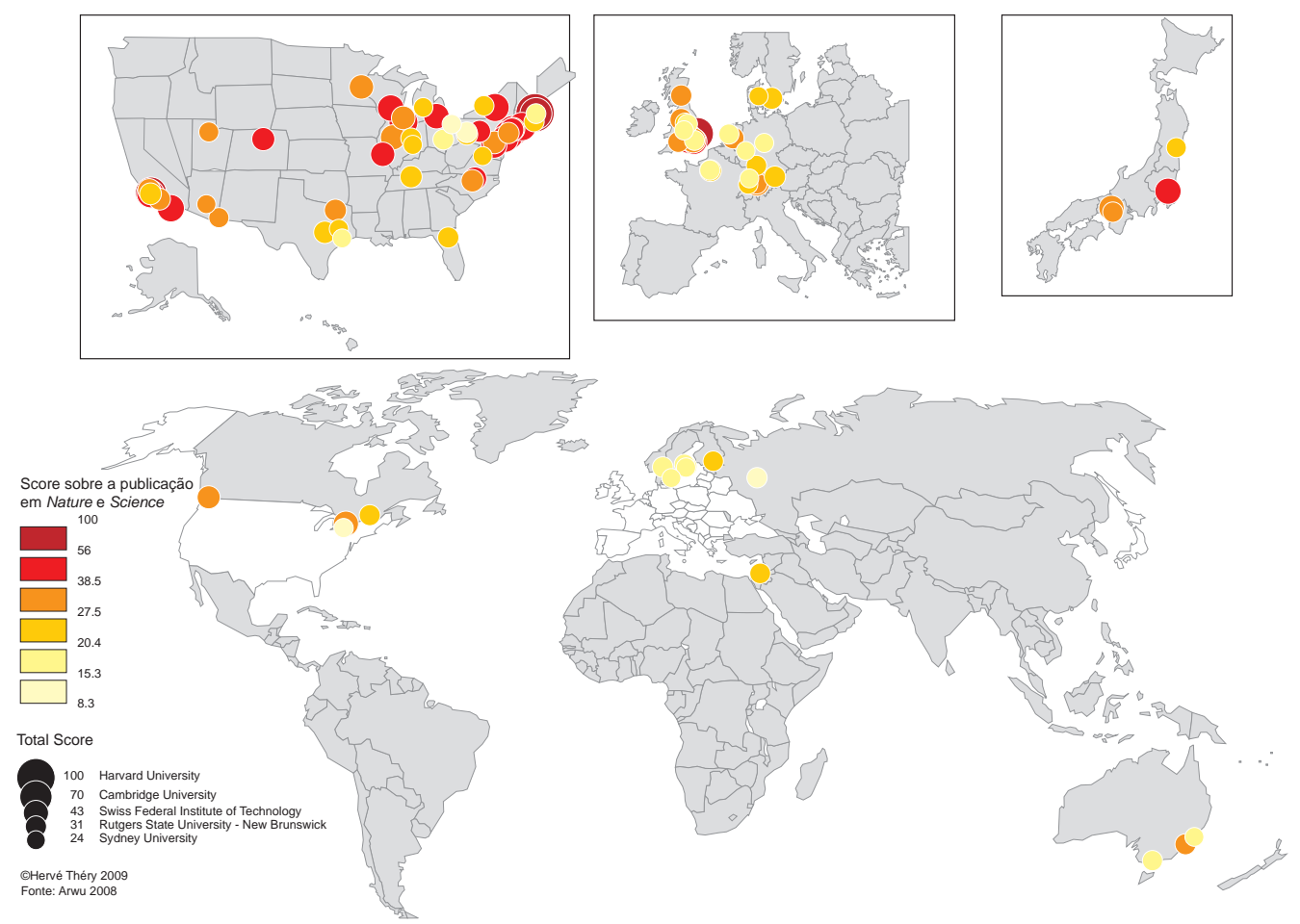

Figura 2 - Publicações em Science e Nature.

\section{Ponderação dos critérios e classificação global}

$\mathrm{Na}$ classificação final, aos pontos obtidos para cada indicador são aplicados os coeficientes da Tabela 1 , para se chegar à nota de cada estabelecimento.

O resultado dessa classificação pode ser tratado em tabela por país, como fazem-no os autores em seu site (Tabela 2).

Esse índice final não sendo calculado pelos autores para todos os estabelecimentos, resolvemos produzir outro, totalizando os pontos obtidos para os seis indicadores (sem ponderação), e, para aumentar os contrastes no mapa (uma licença cartográfica equivalente da exageração das alturas em um blocodiagrama), levamos o total ao quadrado.

A concentração das 500 universidades classificadas pelo Arwu em algumas regiões do mundo é de tal grandeza que foi necessário acrescentar ao mapamúndi três zooms para detalhar a situação dos Estados Unidos, da Europa e do Japão. Dentro dos Estados Unidos, distingue-se claramente um bloco compacto 
que vai da Nova Inglaterra aos Grandes Lagos, seguido pela Califórnia e - em menor escala - pelo Texas e pela Flórida. Na Europa, a concentração das principais universidades reconstitui praticamente a "dorsal europeia" ou "banana azul", ${ }^{3}$ do norte da Inglaterra à Lombardia. No Japão, corresponde à terceira grande megalópole mundial, cuja predominância é assim confirmada pela geografia das grandes universidades.

Tabela 1 - Os critérios do ranking Arwu

\begin{tabular}{l|l|c|c}
\hline \multicolumn{1}{c|}{ Critérios } & \multicolumn{1}{|c|}{ Indicador } & Código & Peso \\
\hline Qualidade da educação & Ex-alunos com medalhas Fields e Prêmios Nobel & Alumi & $10 \%$ \\
\hline $\begin{array}{l}\text { Qualidade do corpo } \\
\text { docente }\end{array}$ & $\begin{array}{l}\text { Funcionários com medalhas Fields e Prêmios } \\
\text { Nobel }\end{array}$ & Award & $20 \%$ \\
\cline { 2 - 4 } & $\begin{array}{l}\text { Pesquisadores mais citados em 21 campos } \\
\text { disciplinares }\end{array}$ & $\mathrm{HiCi}$ & $20 \%$ \\
\hline $\begin{array}{l}\text { Resultados das } \\
\text { pesquisas }\end{array}$ & Artigos publicados em Nature e Science * & $\mathrm{N \& S}$ & $20 \%$ \\
\cline { 2 - 4 } & $\begin{array}{l}\text { Artigos citados em Science Citation Index- } \\
\text { expanded e Social Science Citation Index }\end{array}$ & $\mathrm{SCl}$ & $20 \%$ \\
\hline $\begin{array}{l}\text { Tamanho do } \\
\text { estabelecimento }\end{array}$ & $\begin{array}{l}\text { Resultados dos outros índices divididos pelo } \\
\text { número de funcionários do estabelecimento }\end{array}$ & Size & $10 \%$ \\
\hline
\end{tabular}

* Para estabelecimentos especializados nas ciências humanas e nas ciências sociais tais como o London School of Economics, o criténrio "artigos publicados em Nature e Science" não é considerado, e seu peso é afetado a outros indicadores.

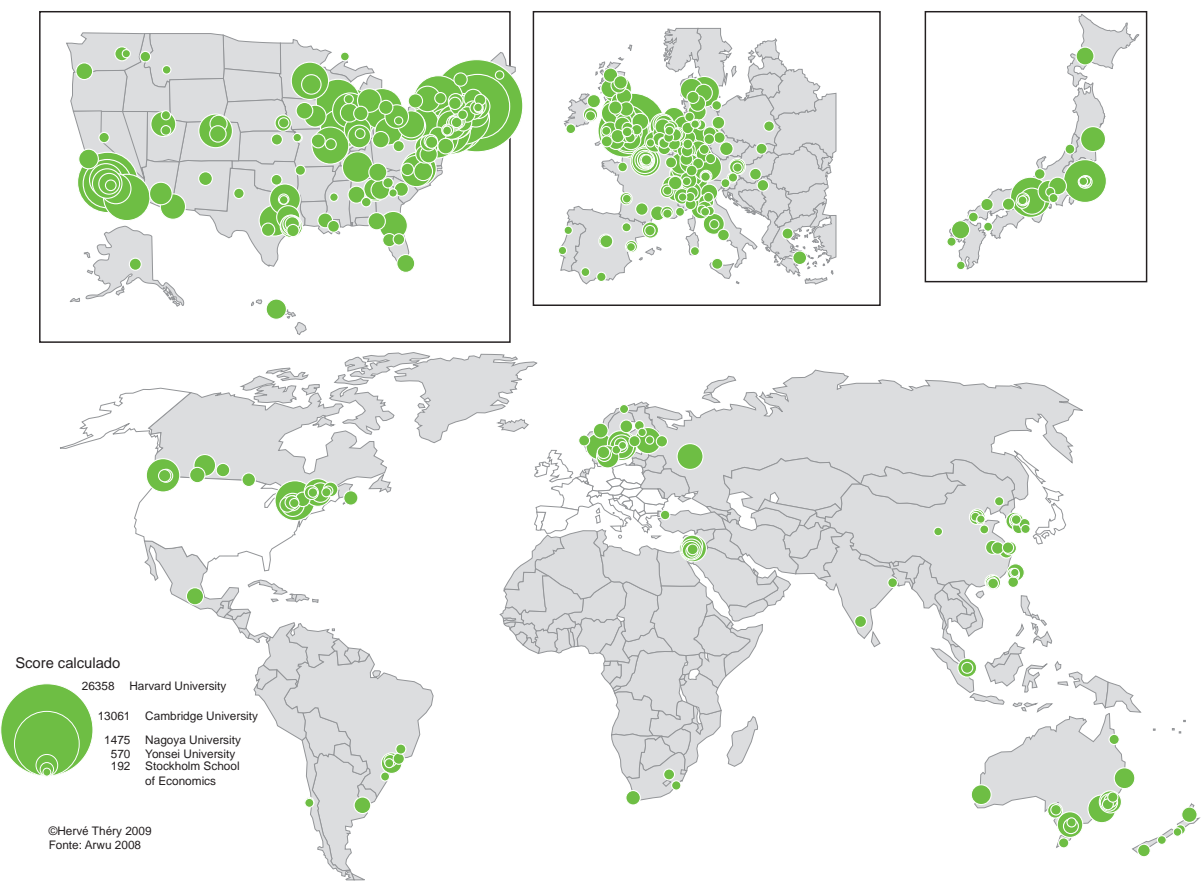

Figura 3 - O ranking Arwu (“de Xangai”) 2008. 
Tabela 2 - As universidades do ranking Arwu por país

\begin{tabular}{c|c|c|c|c}
\hline País & $\begin{array}{c}\text { \% das 100 } \\
\text { primeiras }\end{array}$ & $\begin{array}{c}\text { \% das 500 } \\
\text { primeiras }\end{array}$ & $\begin{array}{c}\text { \% do } \\
\text { PNB mundial }\end{array}$ & $\begin{array}{c}\% \text { da } \\
\text { população }\end{array}$ \\
\hline Estados Unidos & 54,0 & 31,6 & 27,2 & 4,6 \\
\hline Reino Unido & 11,0 & 8,3 & 4,9 & 0,9 \\
\hline Alemanha & 6,0 & 8,0 & 6,0 & 1,3 \\
\hline Japão & 4,0 & 6,2 & 9,0 & 2,0 \\
\hline Canadá & 4,0 & 4,2 & 2,6 & 0,5 \\
\hline Suécia & 4,0 & 2,2 & 0,8 & 0,1 \\
\hline França & 3,0 & 4,6 & 4,6 & 0,9 \\
\hline Austrália & 3,0 & 3,0 & 1,6 & 0,3 \\
\hline Suíça & 3,0 & 1,6 & 0,8 & 0,1 \\
\hline Países Baixos & 2,0 & 2,4 & 1,4 & 0,2 \\
\hline Dinamarca & 2,0 & 0,8 & 0,6 & 0,1 \\
\hline Finlândia & 1,0 & 1,2 & 0,4 & 0,1 \\
\hline Israel & 1,0 & 1,2 & 0,3 & 0,1 \\
\hline Noruega & 1,0 & 0,8 & 0,7 & 0,1 \\
\hline Rússia & 1,0 & 0,4 & 2,0 & 2,2 \\
\hline
\end{tabular}

Fonte: Arwu (2008).

Se escolhas gráficas um pouco diferentes tivessem juntado o Canadá aos Estados Unidos e a Europa do Norte ao resto da União Europeia, o corpo principal do mapa-múndi apareceria quase vazio: nele se encontrariam apenas alguns estabelecimentos latino-americanos (México, São Paulo e Buenos Aires), as universidades australianas e chinesas, uma na Índia, uma em Cingapura e uma única universidade africana, na Cidade do Cabo.

\section{Críticas feitas à classificação Arwu e respostas dos seus autores}

Philippe Mahrer, diretor do Collège des Ingénieurs, na França, critica essa classificação: países que dispõem de muitas escolas ou universidades de excelência mas com poucos alunos são mal classificados, e a Arwu ignora universidades, centros de pesquisa ou escolas que trabalham em rede, como é cada vez mais o que acontece na Europa. Este último ponto é igualmente denunciado pelo titular do Prêmio Nobel de Física 2007, Albert Fert. Da mesma maneira Edouard Mathieu (Head of the Benchmarking Center, Invest in France Agency), nota que apenas 11 universidades conseguem 50 pontos na rubrica alumni (exalunos), contra 305 que não conseguem nenhum ponto nesse quesito, e 372 nenhum ponto para os seus professores, esse critério valendo apenas para as dez ou doze primeiras. De acordo com Violaine Leloup, da embaixada da França em 
Tabela 3 - Os 20 primeiros do ranking Arwu 2008

\begin{tabular}{|c|c|c|}
\hline & Instituição & $\begin{array}{c}\text { Escore } \\
\text { total }\end{array}$ \\
\hline 1 & Harvard University & 100 \\
\hline 2 & Stanford University & 73,7 \\
\hline 3 & University of California - Berkeley & 71,4 \\
\hline 4 & University of Cambridge & 70,4 \\
\hline 5 & Massachusetts Institute of Technology (MIT) & 69,6 \\
\hline 6 & California Institute of Technology & 65,4 \\
\hline 7 & Columbia University & 62,5 \\
\hline 8 & Princeton University & 58,9 \\
\hline 9 & University of Chicago & 57,1 \\
\hline 10 & Oxford University & 56,8 \\
\hline 11 & Yale University & 54,9 \\
\hline 12 & Cornell University & 54,1 \\
\hline 13 & University of California - Los Angeles & 52,4 \\
\hline 14 & University of California - San Diego & 50,3 \\
\hline 15 & University of Pennsylvania & 49,0 \\
\hline 16 & University of Washington - Seattle & 48,3 \\
\hline 17 & University of Wisconsin - Madison & 47,4 \\
\hline 18 & University of California - San Francisco & 46,6 \\
\hline 19 & Tokyo University & 46,4 \\
\hline 20 & Johns Hopkins University & 45,5 \\
\hline
\end{tabular}

Fonte: Arwu (2008).

em Pequim, nesses critérios não aparecem orientações importantes como a formação superior dos executivos, engenheiros e gestores, ou as ciências humanas e sociais. Os setores da engenharia e da gestão, a abertura social e a inovação tecnológica são pouco considerados nos critérios da classificação.

Uma das objeções mais frequentes é de duvidar da possibilidade de medir os resultados das universidades por números. Os autores respondem, não sem humor, a que os professores o fazem constantemente, dando notas aos seus estudantes... É necessário apenas, tanto para as universidades como para os estudantes, recordar que não se devem tomar essas notas como referências absolutas.

O lugar das ciências sociais e humanas é claramente subestimado nessa classificação, os próprios autores o reconhecem, mas eles confessam não ter encontrado, para esses campos científicos, critérios que correspondam às exigências que tinham fixado: medidas universalmente reconhecidas como válidas e 
livremente acessíveis na internet. Em especial, o fato de não poder dispor de uma classificação dos livros, um dos principais meios de expressão dessas ciências, prejudicou a sua inclusão correta na classificação.

Outro fator de distorção é que o inglês tornou-se, na maior parte dos campos científicos, a língua internacional e que os universitários do mundo anglófono são muito mais integrados ao circuito internacional que os seus homólogos dos outros blocos culturais. Consequentemente, essa classificação das universidades favoreceu claramente os países para os quais o inglês é a língua materna.

Uma das perguntas mais espinhosas é determinar à qual universidade atribuir as publicações de cada pesquisador: o princípio usado foi basear-se nas declarações dos próprios autores, na assinatura dos artigos. Na nota explicativa que acompanha a classificação, os autores mencionam explicitamente o caso dos pesquisadores do CNRS francês, cujas publicações só podem ser ponderadas se eles forem ligados a uma universidade, mencionada ao assinarem o artigo. ${ }^{4}$

Além das críticas que lhe foram feitas, outra maneira de responder à classificação "de Xangai" foi construir outras, fundamentadas em outros critérios. Entre todas aquelas que emergiram nos últimos anos, escolhemos três, criadas por instituições de três países diferentes (França, Grã-Bretanha e Espanha) e com bases bem diferentes.

\section{A classificação da École des Mines de Paris}

Em resposta a essa classificação, em que as "grandes escolas" francesas aparecem pouco, a École des Mines de Paris (Mines ParisTech) realizou uma "Classificação internacional profissional dos estabelecimentos de ensino superior" (http://www.mines-paristech.fr/Actualites/PR/), estabelecida a partir do número de ex-alunos que figuram entre os líderes das 500 maiores empresas mundiais.

O princípio é claro:

[...] escolhemos um critério simples, não declarativo e verificável: o número de ex-alunos que ocupam o posto de principal executivo (Chief Executive Officer - CEO - ou equivalente) em uma das 500 maiores empresas internacionais, na data e com os elementos (empresas e líderes) da classificação "Fortune Global 500" estabelecida pela revista Fortune em 2007 a partir do faturamento publicado pelas empresas. Este critério quer ser o equivalente, para as empresas, do critério "ex-alunos que ganharam o Prêmio Nobel ou a medalha Fields" utilizado pela classificação Arwu, o número de pessoas sendo da mesma ordem de grandeza. Mas ao contrário da classificação de Xangai, este critério permite insistir no desempenho da formação dada nos estabelecimentos de ensino superior, em vez do desempenho da pesquisa destes estabelecimentos.

Os pontos obtidos, para cada universidade ou escola, pelo conjunto dos 500 líderes são acumulados, o que permite classificá-las. Por último, para melhorar a legibilidade do resultado, e assim como para a classificação de Xangai, o valor 100 é afetado ao estabelecimento que obteve maior número de pontos, o dos outros estabelecimentos sendo definido como um percentual desse desempenho. 
Tabela 4 - Os vinte primeiros do ranking 2008 da École Nationale des Mines

\begin{tabular}{|c|c|c|}
\hline $\begin{array}{l}\text { Ranking } \\
\text { mundial }\end{array}$ & Instituição & Pontos \\
\hline 1 & Tokyo University & 100 \\
\hline 2 & Harvard University & 89 \\
\hline 3 & Stanford University & 57 \\
\hline 4 & Keio University & 48 \\
\hline 5 & University of Pennsylvania & 39 \\
\hline 6 & Waseda University & 36 \\
\hline 7 & HEC & 35 \\
\hline 8 & Kyoto University & 30 \\
\hline 8 & University of Oxford & 30 \\
\hline 10 & ENA & 29 \\
\hline 11 & Institut d'Études Politiques Paris & 26 \\
\hline 12 & Yale University & 26 \\
\hline 13 & Massachusetts Institute of Technology (MIT) & 24 \\
\hline 13 & Osaka University & 24 \\
\hline 15 & École Polytechnique & 23 \\
\hline 16 & Seoul National University & 23 \\
\hline 17 & University Muenster & 22 \\
\hline 18 & Fordham University & 21 \\
\hline 18 & Pennsylvania State University - University Park & 21 \\
\hline 20 & Duke University & 20 \\
\hline
\end{tabular}

Fonte: Mines ParisTech (2008).

É óbvio que os resultados de um país são fortemente correlacionados ao número de empresas desse país representadas na Fortune Global 500, as empresas sendo geralmente dirigidas por pessoas que estudaram em seu país de origem. Como os autores da Arwu, os autores dessa classificação respondem a algumas das objeções que lhes foram feitas, por exemplo: "Os CEO estudaram há cerca de trinta anos, é o passado dos estabelecimentos que é avaliado? Exatamente [...] como para os Prêmios Nobel...".

Se algumas diferenças aparecem nas hierarquias, como o primeiro lugar obtido pela Universidade de Tóquio ou o aparecimento no topo da classificação das escolas francesas (HEC, ENA ou École Polytechnique ${ }^{6}$ ), a imagem de conjunto que aparece no mapa continua praticamente a mesma. Apenas nota-se que as universidades da Califórnia se destacam menos, que Paris e Tóquio obtêm melhores resultados e que um número um pouco maior de universidades chinesas e brasileiras (a Mackenzie, por exemplo) aparecem, por formar os executivos de seu país. 


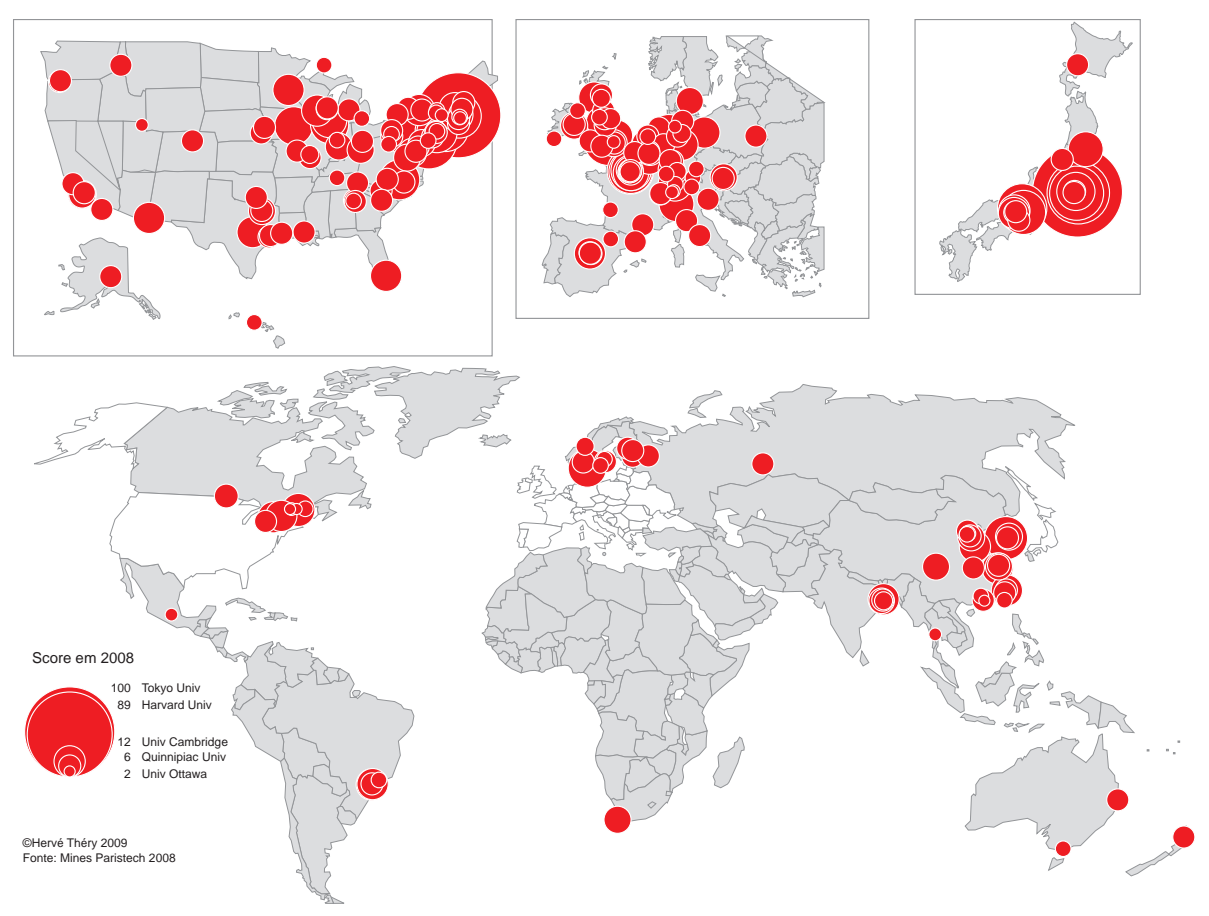

Figura 4-O ranking da École Nationale des Mines.

\section{A classificação do Times Higher Education}

A classificação estabelecida por esse jornal britânico, referência reconhecida nos meios universitários, visava levar em conta as diferentes funções da universidade (especialmente a formação dos estudantes), e não somente os resultados da pesquisa feita nelas. Cinco critérios foram escolhidos, que vão desde a avaliação feita por outros universitários a respeito de seus concorrentes (peer review) ao julgamento dos empregadores, passando pelo número e pela qualidade das citações dos trabalhos produzidos nessa universidade e pelo número de estudantes por docente.

AVALIAÇÃo "PElos PARES". A avaliação das universidades "pelos pares" é o critério principal, medida graças a uma pesquisa realizada na internet (no mundo inteiro), junto aos universitários que opinam - obviamente, por razões éticas sobre estabelecimentos outros que o seu próprio. O resultado é um pouco mais homogêneo que os anteriores, mas, com exceção da boa classificação das universidades australianas, as hierarquias continuam mais ou menos as mesmas, com uma nítida predominância da Califórnia e do nordeste dos Estados Unidos.

AVAliaÇÃo PElos EMPREgadores. Também fundada numa pesquisa feita na internet, essa avaliação foi obtida junto aos empregadores, aos quais foi pedido que avaliassem a qualidade da formação dada aos seus funcionários pelos estabelecimentos universitários onde obtiveram o seu diploma. As universidades britânicas e australianas obtêm aqui bons resultados, bem como Paris e Barcelona. 
Tabela 5 - Os critérios do Times Higher Education

\begin{tabular}{l|l|c}
\hline \multicolumn{1}{c|}{ Indicador } & \multicolumn{1}{|c}{ Explicação } & Peso \\
\hline Avaliação pelos pares & $\begin{array}{l}\text { Pontos obtidos na pesquisa junto aos pares } \\
\text { (dividido em cinco campos científicos), 6.354 } \\
\text { respostas em 2008. }\end{array}$ & $40 \%$ \\
\hline $\begin{array}{l}\text { Avaliação pelos } \\
\text { empregadores }\end{array}$ & $\begin{array}{l}\text { Pontos obtidos na pesquisa junto aos } \\
\text { empregadores, 2.339 respostas em 2008. }\end{array}$ & $10 \%$ \\
\hline $\begin{array}{l}\text { Relação docentes/ } \\
\text { discentes }\end{array}$ & $\begin{array}{l}\text { Relação entre os números de estudantes e de } \\
\text { professores. }\end{array}$ & $20 \%$ \\
\hline Citações por faculdade & $\begin{array}{l}\text { De números citações divididas pelo número de } \\
\text { pesquisadores. }\end{array}$ & $20 \%$ \\
\hline $\begin{array}{l}\text { Internacionalização do } \\
\text { corpo docente }\end{array}$ & Proporção de professores estrangeiros. & $5 \%$ \\
\hline $\begin{array}{l}\text { Internacionalização dos } \\
\text { estudantes }\end{array}$ & Proporção de estudantes estrangeiros. & $5 \%$ \\
\hline
\end{tabular}

Tabela 6 - Os vinte primeiros no ranking do Times Higher Education

\begin{tabular}{c|c|l|c}
\hline $\begin{array}{c}\text { Ranking } \\
\mathbf{2 0 0 8}\end{array}$ & $\begin{array}{c}\text { Ranking } \\
\mathbf{2 0 0 7}\end{array}$ & \multicolumn{1}{|c}{ Instituição } & $\begin{array}{c}\text { Escore } \\
\text { total }\end{array}$ \\
\hline 1 & 1 & Harvard University & 100 \\
\hline 2 & 2 & Yale University & 99,8 \\
\hline 3 & 2 & University of Cambridge & 99,5 \\
\hline 4 & 2 & University of Oxford & 98,9 \\
\hline 5 & 7 & California Institute of Technology & 98,6 \\
\hline 6 & 5 & Imperial College London & 98,4 \\
\hline 7 & 9 & University College London & 98,1 \\
\hline 8 & 7 & University of Chicago & 98 \\
\hline 9 & 10 & Massachusetts Institute of Technology & 96,7 \\
\hline 10 & 11 & Columbia University & 96,3 \\
\hline 11 & 14 & University of Pennsylvania & 96,1 \\
\hline 12 & 6 & Princeton University & 95,7 \\
\hline 13 & 13 & Duke University & 94,4 \\
\hline 13 & 15 & Johns Hopkins University & 94,4 \\
\hline 15 & 20 & Cornell University & 94,3 \\
\hline 16 & 16 & Australian National University & 92 \\
\hline 17 & 19 & Stanford University & 91,2 \\
\hline 18 & 38 & University of Michigan & 91 \\
\hline 19 & 17 & University of Tokyo & 90,7 \\
\hline 20 & 12 & McGill University & \\
\hline
\end{tabular}

Fonte: Times Higher Education (2008). 

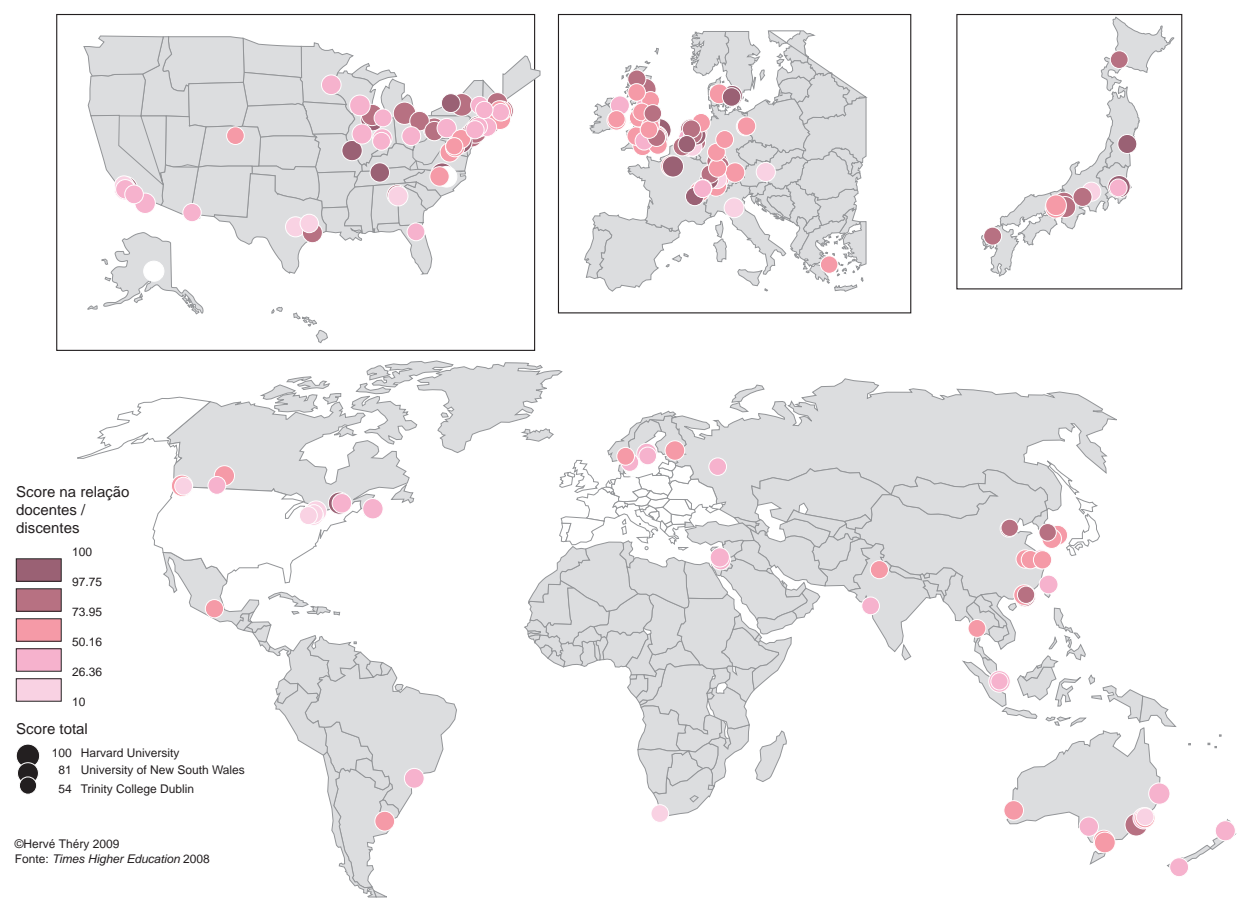

Figura 5 - Relação docentes/discentes do Times Higher Education.

CitAções. Para medir os resultados da pesquisa, levou-se em conta, como na classificação Arwu, o número de citações dos autores que trabalham em cada universidade, utilizando-se da base de dados Scopus, nos últimos cinco anos. O resultado é sensivelmente o mesmo, com uma esmagadora predominância dos países anglófonos, especialmente dos Estados Unidos.

DOCENTES POR ALUNO. Para não limitar-se aos resultados da pesquisa, e levar em conta a forma como as universidades seguem, no dia a dia, os progressos de seus estudantes, a revista optou por um critério simples e facilmente mensurável, a relação entre os números de estudantes e de professores. Ao supor-se que quanto mais alto o número dos segundos em relação aos primeiros, mais elevada a atenção dada a eles, é uma maneira de medir a qualidade da formação dada. Paris obtém aqui bons resultados, especialmente graças às "grandes escolas" onde a admissão seletiva reduz sensivelmente o número de estudantes em relação às universidades, abertas sem vestibular aos alunos que passaram no baccalauréat, o exame de conclusão do ensino médio.

\section{Outros critérios e classificação final}

Outro critério que foi levado em conta foi a abertura de cada universidade em relação ao mundo externo, medido pelo número de estudantes e professores que provêm de outros países. De certa maneira, é também uma medida da atração de cada estabelecimento fora dos limites de seu próprio país. Os critérios foram combinados para obter um resultado global, cujas ponderações estão mencionadas na Tabela 5 . 
A imagem global confirma mais uma vez a predominância das três megalópoles, a americana (estendida até os Grandes Lagos e reforçada pelo Sun Belt, da Califórnia até a Flórida), a europeia e a japonesa, às quais basta acrescentar o Canadá, a Europa do Norte, a China e a Austrália para obter o essencial da geografia das grandes universidades mundiais.

\section{O Webometrics Ranking of World's Universities}

O Webometrics Ranking of World Universities é uma iniciativa do laboratório Cybermetrics, um grupo de pesquisa que pertence ao Consejo Superior de Investigaciones Científicas (CSIC), a maior instituição pública de pesquisa da Espanha, com 126 centros e institutos em todo o país, o qual integra o Ministério da Educação. O laboratório tem por objetivo a análise quantitativa da internet e concebeu indicadores que visam medir a atividade científica na web. O objetivo original da classificação era favorecer a publicação na web - e não classificar os estabelecimentos -, mas esses indicadores dão sinalizações úteis porque não são baseadas no número de visitas ou na concepção das páginas, mas na visibilidade global das universidades na rede. Uma forte presença na web, fornecendo descrições exatas da estrutura e das atividades da universidade, pode atrair novos estudantes e professores do mundo inteiro, e o número de consultas recebidas por um site é uma medida da visibilidade e do impacto de seu conteúdo. Mais de quinze mil universidades foram avaliadas (contra três mil pelo Arwu), um pouco mais de quatro mil na Europa, 3.500 na América do Norte, 3.600 na Ásia, 2.800 na América Latina, 500 na África e 100 na Oceania.

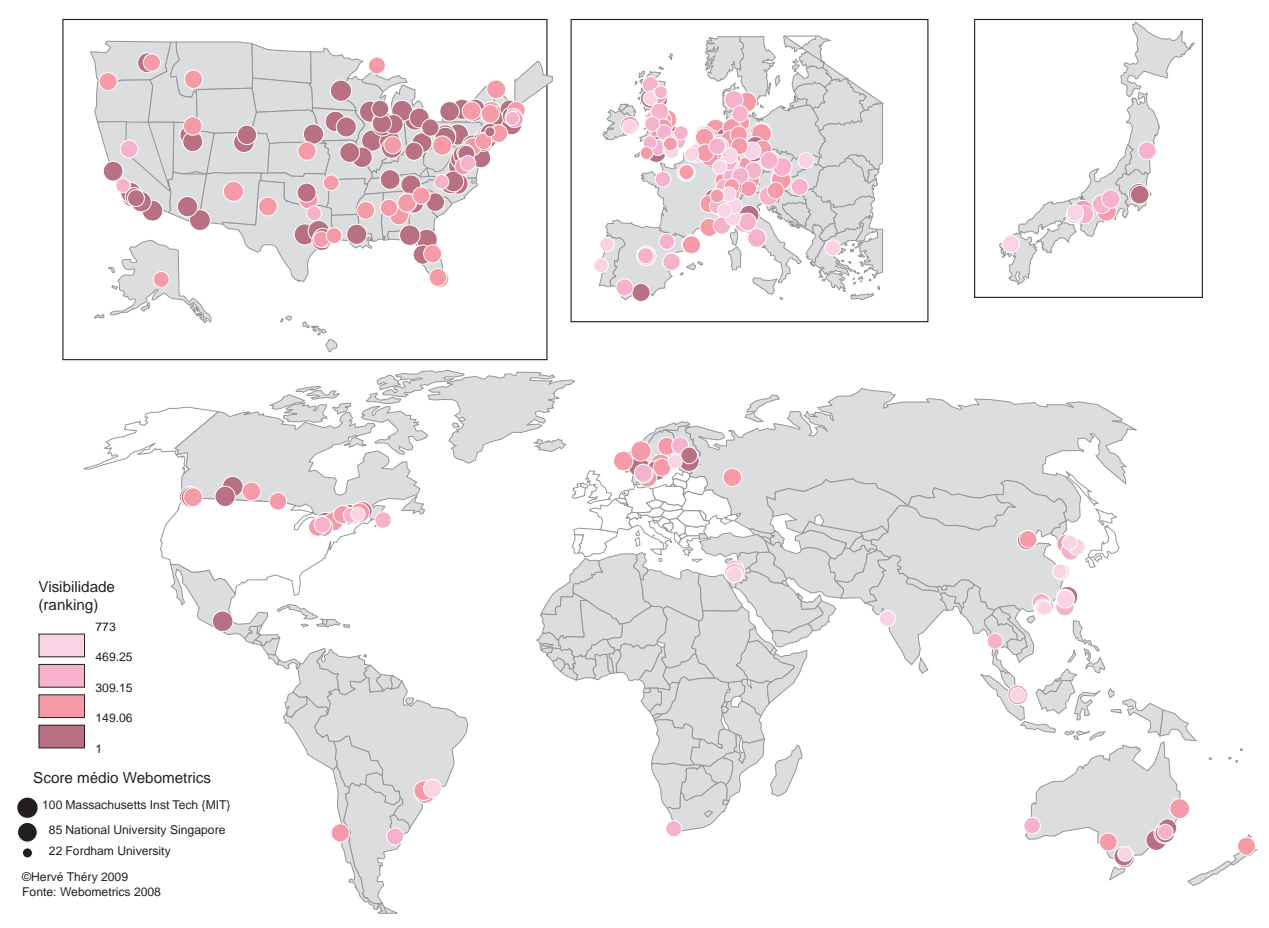

Figura 6 - A "visibilidade" no ranking Webometrics. 
Tabela 7 - Os vinte primeiros do ranking Webometrics 2008

\begin{tabular}{|c|c|c|}
\hline $\begin{array}{l}\text { Ranking } \\
\text { mundial }\end{array}$ & Instituição & Site \\
\hline 1 & Massachusetts Institute of Technology & mit.edu \\
\hline 2 & Harvard University & harvard.edu \\
\hline 3 & Stanford University & stanford.edu \\
\hline 4 & University of California Berkeley & berkeley.edu \\
\hline 5 & Cornell University & cornell.edu \\
\hline 6 & University of Wisconsin Madison & wisc.edu \\
\hline 7 & University of Minnesota & umn.edu \\
\hline 8 & California Institute of Technology & caltech.edu \\
\hline 9 & University of Illinois Urbana Champaign & uiuc.edu \\
\hline 10 & University of Michigan & umich.edu \\
\hline 11 & University of Texas Austin & utexas.edu \\
\hline 12 & University of Washington & washington.edu \\
\hline 13 & University of Chicago & uchicago.edu \\
\hline 14 & Carnegie Mellon University & cmu.edu \\
\hline 15 & University of Pennsylvania & upenn.edu \\
\hline 16 & Columbia University New York & columbia.edu \\
\hline 17 & Texas A\&M University & tamu.edu \\
\hline 18 & University of Maryland & umd.edu \\
\hline 19 & University of California Los Angeles & ucla.edu \\
\hline 20 & Purdue University & purdue.edu \\
\hline
\end{tabular}

Fonte: Webometrics Ranking of World Universities (2008).

Quatro indicadores foram obtidos partir dos resultados quantitativos fornecidos principais pelos motores de pesquisa: dimensão, visibilidade, qualidade das páginas e conteúdo científico.

A dimensão é o número de páginas recolhidas por quatro motores de pesquisa: Google, Yahoo, Live Search e Exalead.

A visibilidade é o número de relações externas únicas recebidas (inlinks) por um site, comunicado aos autores da pesquisa (confidencialmente) por Yahoo, Live Search e Exalead.

A qualidade das páginas é medida pelo tipo de arquivo. Após avaliação de sua importância para atividades educativas, contaram-se as páginas nos formatos informáticos seguintes: Adobe Acrobat (pdf), Adobe PostScript (ps), Microsoft Word (doc) e Microsoft Powerpoint (ppt). Esses dados foram extraídos utilizando Google, Yahoo Search, Live Search e Exalead. Nesse caso, a Europa continental parece ter feito esforços importantes, chegando ao nível das universidades do norte dos Estados Unidos e de algumas do sul. 
A fonte sobre o conteúdo cientifico é o serviço especializado de Google, Google Scholar, que fornece o número de artigos e citações para cada campo científico. Dessa vez, o nordeste dos Estados Unidos e a Califórnia voltam a ser predominantes.

Os quatro indicadores são combinados com uma ponderação de $50 \%$ para a visibilidade, $20 \%$ para a dimensão, $15 \%$ para a qualidade das páginas e $15 \%$ para o conteúdo científico.

A lista dos vinte primeiros estabelecimentos é composta inteiramente de universidades dos Estados Unidos, que não deixam nenhum lugar às concorrentes estrangeiras. O mapa sublinha, pela quarta vez, a predominância das regiões já mencionadas. No máximo, pode-se dizer que as pequenas e médias universidades chegam a compensar, nesse campo de atividade, a desproporção dos meios disponíveis em relação às grandes universidades, e que o seu site pode ser de qualidade comparável.

\section{Uma classificação das classificações?}

No fim desta análise das quatro classificações, um tratamento estatístico ajuda a revelar as diferenças e a construir uma tipologia das universidades mundiais. Usamos uma classificação ascendente hierárquica (clusters) dos estabelecimentos (universidades e escolas) presentes nas quatro classificações, ou seja, 96 no total. Fazer parte dessa lista curta já é um critério em si, já que é necessário para ficar nela um reconhecimento quantificado da sua produção científica (Arwu), da sua capacidade de produzir líderes de grandes grupos econômicos (Mines ParisTech), da qualidade do seu ensino (Times Higher Education) e a sua presença na internet (Webometrics). Os critérios escolhidos aparecem na Figura 7 e permitem construir perfis que agrupam as universidades semelhantes perante esses critérios. Pelo seu princípio mesmo, a análise de clusters as agrupa em função do desvio à média, para cada variável, em relação ao grupo avaliado, quer acima a média (barras à direita do eixo vertical), quer abaixo de esta (barras à esquerda).

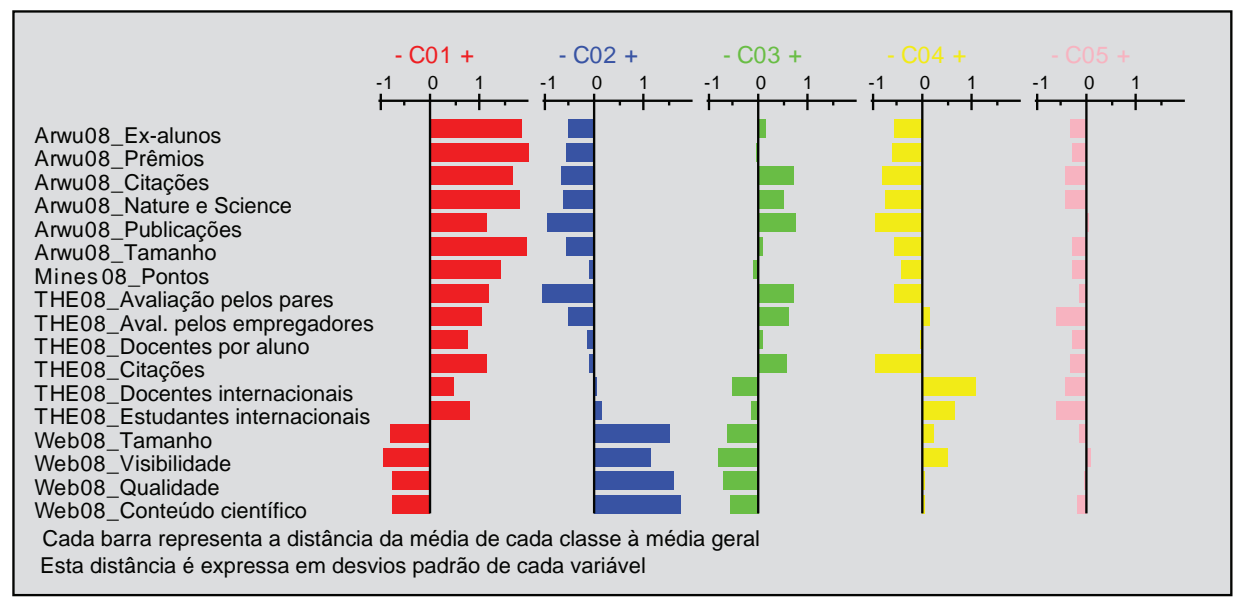

Figura 7 - Critérios da tipologia. 

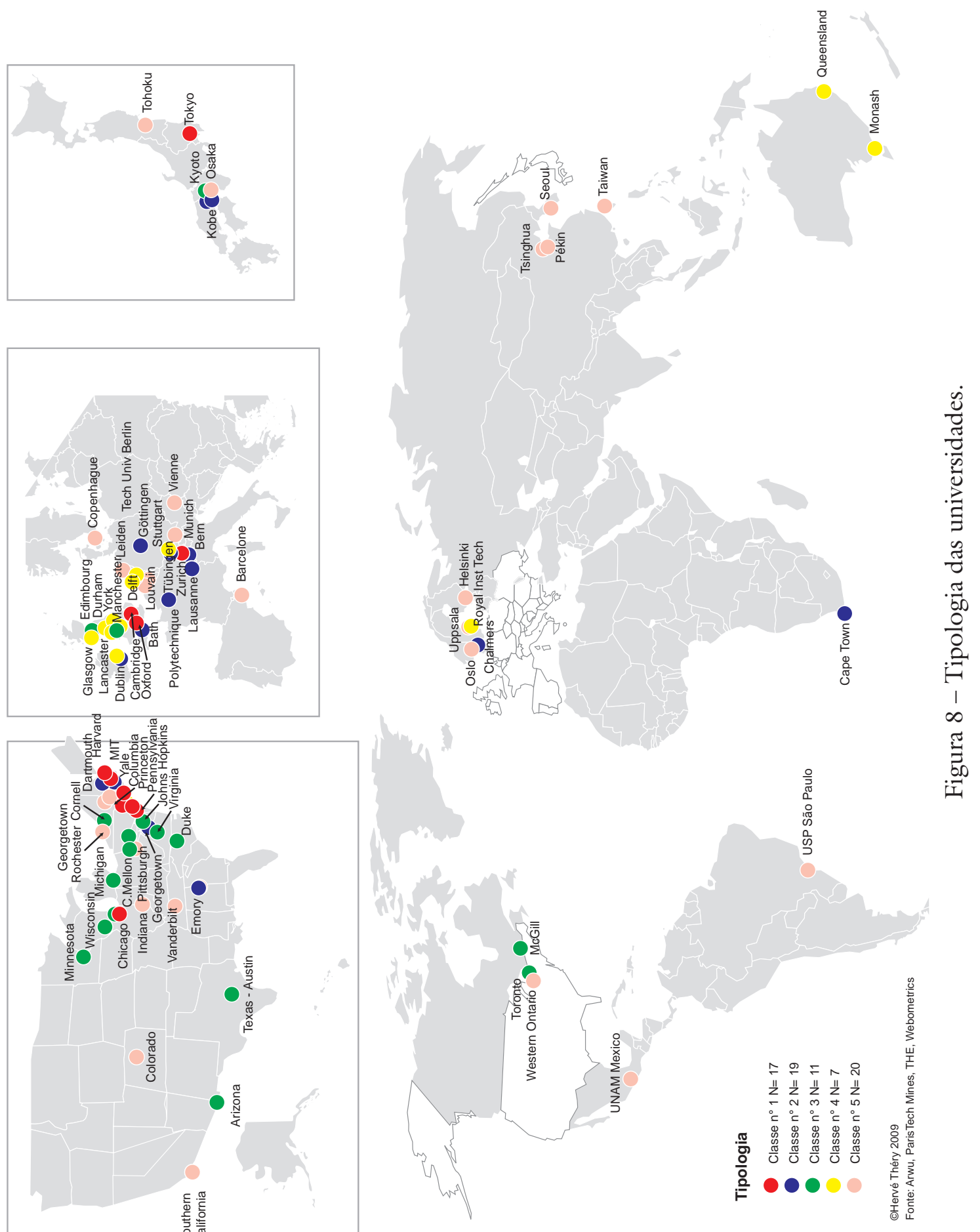
Essa tipologia pode ser interpretada da maneira seguinte:

O GRUPO N. 1 (círculos vermelhos na Figura 8) é visivelmente o do "bons aluno", os que têm resultados muito superiores à média para todos os critérios (menos a sua presença na web, do qual não têm provavelmente tanto necessidade). São principalmente as universidades da Ivy league ${ }^{7}$ da Nova Inglaterra, mais Chicago, Oxford, Cambridge, Zurique (Instituto Federal de Tecnologia) e Tóquio.

O GRUPO N. 2 (círculos azuis) é, pelo contrário, o que se distingue especialmente pela qualidade - e a frequentação - da sua presença no web. Ele associa universidades um pouco menos conhecidas dos Estados Unidos (Emory, Dartmouth, Georgetown) ou da Europa (Bath, Göttingen, Tübingen, Chalmers), bem como a École Polytechnique e a Universidade de Cape Town, que lhe devem respectivamente a presença dos únicos estabelecimentos franceses e africanos.

O GRUPO N. 3 (círculos verdes) é caracterizado por resultados melhores que a média para as publicações, especialmente em Nature e Science, uma boa avaliação pelos pares (e os empregadores), um grande número de citações dos seus professores nos índices reconhecidos. Ou seja, um perfil mais "acadêmico" e levando a carreiras menos brilhantes - e remuneradores - que o grupo n.l. Agrupa universidades do Middle West e do sul dos Estados Unidos, Mc Gill e Toronto, Quioto, Manchester e Edimburgo.

O GRUPO N. 4 (círculos amarelos) está mais aberto sobre o mundo, ele conta com mais estudantes e professores estrangeiros, os seus sites são abundantes e muito visitados (uma coisa explicando provavelmente a outra): essas universidades australianas, holandesas, irlandesas e sobretudo britânicas (Glagow, Durham, York, Sheffield) souberam tornar-se atrativas por outros meios que as dos Estados Unidos ou do Japão, as quais não aparecem nesse grupo.

O GRUPO N. 5 (círculos cor-de-rosa), finalmente, está próximo da média para todos os critérios, e parecer ser nessa elite mundial, o dos países (China, Brasil, México), e regiões emergentes - do ponto de vista universitário - da Europa (Escandinávia, Espanha, Áustria, Bélgica). Nos Estados Unidos, ele é formado por estabelecimentos menos prestigiosos que os do primeiro grupo, menos presentes na web que o segundo, que publicam menos que o terceiro, menos internacionais que o quarto, mas deve constar nesse grupo porque está presente em todos os domínios. Trata-se um bom desempenho, enquanto outros são ausentes dessa short list por não terem conseguido qualificar-se em um ou outro desses campos de competência: é o caso de toda a Califórnia, com uma única exceção.

\section{Conclusão}

Uma primeira conclusão é que a classificação lançada - com ambições modestas - pelo Institute of Higher Education da Shanghai Jiao Tong University teve, pelo menos, como efeito lançar um vasto debate, e ainda que usos perver- 
sos tenham sido feitos, ele não pode ser criticado por isso: cada um - universidades, ministérios, professores e estudantes - deve saber usar essa classificação e conhecer as suas limitações. O seu sucesso suscitou outras iniciativas, as que foram analisadas aqui e outras mais (ver na bibliografia classificações especializadas para os estudantes pretos, hispânicos, ativistas etc.). Com os concorrentes que fez nascer, levou as universidades do mundo inteiro a melhorar, se não seu "desempenho", pelo menos a sua apresentação ao mundo externo, e levou muitos parceiros da comunidade educativa a interrogar-se sobre os reais propósitos do ensino superior, sobre as maneiras de medir os seus sucessos, sobre os diferentes sistemas mundiais.

Quando se examinam os resultados dessas classificações que ambicionam ser as concorrentes da primeira, a de Xangai, aparecem nitidamente as suas convergências. As imagens construídas projetando - sem nenhum a priori- os seus resultados em mapas são muito semelhantes. Os poucos desvios são geralmente ligados às escolhas feitas pelos seus promotores para se diferenciar - sem grande sucesso, desse ponto de vista - dos seus antecessores. As imagens globais - marcadas pela concentração dos "bons” estabelecimentos nas três megalópoles e por sua escassez nos outros lugares - são muito semelhantes nos quatro casos, e a análise cartográfica confirma essas semelhanças de maneira incontestável. Poderse-ia então pensar que não adianta criticar as insuficiências da classificação de Xangai, já que as outras, embora construídas com critérios diferentes, conduzem aos mesmos resultados. Não seria completamente justo, no entanto, e a tipologia tirada da análise estatística mostra-o. Mesmo nesse contexto de dominação das megalópoles, estratégias e situações alternativas aparecem. Os mapas indicam associações inéditas, fundadas sobre semelhanças que não apareciam nos rankings. Ao lado dos "bons alunos”, outras universidades distinguem-se graças a um perfil mais "acadêmico", ou à qualidade da sua presença na web, ou à sua abertura sobre o mundo. Além disso, uma nova geração de regiões e de países "emergentes" consegue entrar no grupo de cabeça, anunciando - talvez? - futuros equilíbrios. É uma tendência saudável porque, nesse domínio como em muitos outros, os esforços que levam a mais variedade, mais pluralismo e mais pluricentrismo são bem-vindos.

\section{Notas}

1 "by their research performance based on internationally comparable data that everyone could check."

2 Utilizamos, para o primeiro tratamento cartográfico dos dado, os softwares Cartes et Données e Philcarto. O primeiro é oferecido a preço reduzido aos estudantes e professores (http://www.articque.com/guide-metiers/secteur-education-recherche.html), o segundo está disponível gratuitamente no endereço http://philgeo.club.fr

3 Usando a expressão de Roger Brunet, disponível em: <http://www.mgm.fr/ARECLUS/page_auteurs/Brunetl4.html>. 
4 No caso do autor deste artigo, ele deveria mencionar que o Credal, onde trabalha, é uma unidade mista do CNRS com a Université Sorbonne Nouvelle Paris III. Mas o autor resolveu mencionar - e dar pontos - à USP, que o acolhe como professor visitante.

5 Sistema paralelo às universidades onde são formadas as elites francesas, nos campos de negócios, engenharia, administração pública, ensino superior etc.

6 Hautes Études Commerciales, École Nationale d'Administration e École Polytechnique onde são formados gestores, altos funcionários e engenheiros.

7 A Ivy League é um grupo de oito universidades do nordeste dos Estados Unidos, as mais antigas do país: literalmente significa "liga da hera", planta que cobre as fachadas dos seus prédios históricos. São as instituições de maior prestígio científico, mas a denominação é associada a um certo elitismo e ao establishment.

\section{Referências}

BORDET, A. Classement de Shanghai: branle-le-bas de combat dans les Universités européennes. Cafebabel.com. Disponível em: <http://www.cafebabel.com/fre/article/27444/classement-shanghai-universites-europe.html>. Acesso em: 16 ago. 2009.

CHEIMANOFF, N. (Mines ParisTech): Notre classement constitue un véritable outil de communication à l'international>. Disponível em: <http://www.educpros.fr/detailarticle/h/3ea094lab7/a/nicolas-cheimanoff-mines-paristech-notre-classement-constitue-un-veritable-outil-de-communica.html>. Acesso em: 16 ago. 2009.

FERT, A. Comment le classement de Shanghai désavantage nos Universités. Le Monde, 27 août 2008.

FLORIAN, R. V. Irreproducibility of the results of the Shanghai academic ranking of world universities. Scientometrics, v.72, p.25-32, July 2007.

GINGRAS, Y. La fièvre de l'évaluation de la recherche. Du mauvais usage de faux indicateurs. Notes de recherche du CIRST, mai 2008.

LELOUP, V. Le classement de l'Université de Jiaotong de Shanghai. 30 novembre 2005, mise à jour: 27 avril 2007. Disponível em: <http://www.ambafrance-cn.org/ spip.php?article558\&lang=fr>. Acesso em: 24 ago. 2009.

LIU, N. C. et al. Academic Ranking of World Universities - Methodologies and Problems. Higher Education in Europe, v.30, n.2, 2005a.

Academic Ranking of World Universities, Using Scientometrics: a Comment on the "Fatal Attraction". Scientometrics, v.64, n.1, p.101, 2005 b.

MAHRER, P. Université-Grandes écoles, le vrai poids de la France. Les Échos, 5 déc. 2007.

MERISOTIS, J. P. On the ranking of higher education institutions. Higher Education in Europe, v.27, n.4, p.361, 2002.

OBSERVATOIRE BOIVIGNY. Classement de l'école des Mines: critiques et contrecritiques. Disponível em: <http://www.boivigny.com/Classement-de-l-ecole-des-Mines-critiques-et-contre-critiques_a555.html>. Acesso em: 16 ago. 2009.

RAAN, A. F. J. van. Fatal attraction: ranking of universities by bibliometric methods. Scientometrics, v.62, n.1, p.133, 2005. 
SAISANA, M.; D'HOMBRES, B. Higher Education Rankings: robustness issues and critical assessment. How much confidence can we have in Higher Education Rankings? Rapport EUR 23487 pour l'Union Européenne, 2008.

VAN LEEUWEN, T. N. Testing the validity of the Hirsch-index for research assessment purposes. Research Evaluation, v.17, p.157-60, June 2008.

Fontes dos dados

Classificação Arwu e as suas fontes

ACADEMIC Ranking of World Universities, Institute of Higher Education, Shanghai Jiao Tong University. Disponível em: <http://www.arwu.org/>. Acesso em: 24 ago. 2009.

ARTICLES classés Science Citation Index-expanded et Social Science Citation Index. Disponível em: <http://www.isiknowledge.com>. Acesso em: 24 ago. 2009.

ARTICLES édités dans Nature et Science. Disponível em: <http://www.isiknowledge. com>. Acesso em: 24 ago. 2009.

CHERCHEURS les plus cités. Disponível em: <http://www.isihighlycited.com>. Acesso em: 24 ago. 2009.

LAURÉATS de Prix Nobel. Disponível em: <http://nobelprize.org/nobel_prizes/>. Acesso em: 24 ago. 2009.

MEDAILLES Fields. Disponível em: <http://www.mathunion.org/general/prizes/fields/prizewinners/>. Acesso em: 24 ago. 2009.

\section{Outras classificações}

CLASSEMENT international professionnel des établissements d'enseignement supérieur. Disponível em: <http://www.mines-paristech.fr/Actualites/PR/>. Acesso em: 24 ago. 2009.

FORTUNE Global 500. Disponível em: <http://money.cnn.com/magazines/fortune/ global500/2009/>. Acesso em: 24 ago. 2009.

RANKING of World Universities in the Web. Disponível em: <http://www.webometrics.info>. Acesso em: 24 ago. 2009.

THE TIMES Higher Education World University Ranking. Disponível em: <http:// www.timeshighereducation.co.uk/hybrid .asp? typeCode $=41$ 6\&pubCode $=1$ \& navcod e=137>. Acesso em: 24 ago. 2009.

\section{Classificações especializadas}

BLACK ENTERPRISE'S Top Colleges for African Americans. Disponível em: <http:// www.blackenterprise.com/diversity/top-colleges>. Acesso em: 24 ago. 2009.

COST-BENEFIT Analysis of American Law Schools. Disponível em: <http://www.ilrg. com/schools/analysis/>. Acesso em: 24 ago. 2009.

HISPANIC MAGAZINE.COM'S Top 25 Colleges for Latinos. Disponível em: <http:// www.hispaniconline.com/HispanicMag/2008_03/Feature-25Colleges.html>. Acesso em: 24 ago. 2009.

MOTHER JONES Top 10 Activist Schools. Disponível em: <http://www.motherjones. com/politics/2003/09/top-10-activist-campuses >. Acesso em: 24 ago. 2009.

THE 25 BEST distant learning Universities. Disponível em: <http://25best-distant-learning-universities.com/index.htm>. Acesso em: 24 ago. 2009 
RESUMO - A importância dada, no debate internacional sobre Universidades, à classificação dita “de Xangai” nos incitou a analisá-la de perto, a ver como funciona e a transferir os seus resultados sobre um mapa-múndi. Os mapas resultantes revelam uma geografia das grandes universidades mundiais, seus pontos de concentração (as três grandes megalópoles) e seus pontos fracos (o resto do mundo, com raras exceções). Em reação às insuficiências e aos vieses dessa classificação, outras foram elaboradas, três delas são igualmente analisadas e mapeadas.

PALAVRAS-CHAVES: Universidades, Classificações, Megalópoles.

ABSTRACT - The increasing importance, in the international debate on Universities, of the so-called "Shanghai ranking" encouraged us to look at it more closely, to analyse how it works, and to transfer its results to a world map, in order to reveal the geography of world universities, its points of concentration (three three megalopolis) and its weak points (the rest of the world, save a few exceptions). In reaction to the insufficiencies and skews of this classification, other rankings have been devised, three of them are also analysed and mapped.

KEYWORDS: Universities, Classifications, Megalopolis.

Hervé Théry é diretor de pesquisa no CNRS-Credal e professor convidado na Universidade de São Paulo. @ - hthery@aol.com

Recebido em 21.4.2009 e aceito em 17.6.2009. 